\title{
How to best preserve oocytes in female cancer patients exposed to DNA damage inducing therapeutics
}

\author{
K Hutt ${ }^{1}$, JB Kerr ${ }^{2}$, CL Scott ${ }^{3,4}$, JK Findlay ${ }^{1}$ and A Strasser ${ }^{\star, 3,4}$ \\ Cell Death and Differentiation (2013) 20, 967-968; doi:10.1038/cdd.2013.54
}

It has long been recognised that the immature oocytes stored in the ovary as primordial follicles are exquisitely sensitive to DNA damaging anti-cancer treatments, including both radiation and chemotherapy. ${ }^{1}$ This inherent sensitivity often leaves female cancer survivors with a reduced number of oocytes, predisposing them to infertility and premature menopause with associated health problems later in life. ${ }^{1}$ Early detection and improved treatments have led to survival rates as high as $80 \%$ for many cancers and it is becoming increasingly important to consider the fertility and reproductive health of these survivors. ${ }^{2}$ This critical need has inspired a flurry of research activity aimed at understanding the mechanisms by which DNA damage kills oocytes, with the goal of identifying potential targets and strategies to inhibit oocyte death and protect the ovary from damage during anti-cancer treatment. ${ }^{3-6}$

The article by Kim et al..$^{7}$ in this issue of Cell Death and Differentiation explores the mechanisms of oocyte death following exposure to the DNA damage inducing platinumbased chemotherapeutic drug, cisplatin. Kim et al. propose that TAp63 is the master regulator of cisplatin-induced oocyte death, exerting control by regulating the expression of its family members p53 and TAp73, as well as the tyrosine kinase c-Abl. According to their model, after transcriptional induction by TAp63, c-Abl post-translationally activates both TAp63, which then triggers transcription of TAp73, and also TAp73. TAp73 then (either directly or indirectly) activates the transcription of Bax (a multi-BH domain proapoptotic member of the Bcl-2 family), thereby leading to oocyte death (Figure 1). Remarkably, the tyrosine kinase inhibitor imatinib was shown to prevent cisplatin-induced oocyte death in vitro, and in shortterm (14 days) transplantation studies in vivo, possibly by preventing c-Abl-mediated phosphorylation (and consequent activation) of TAp73, although this was not demonstrated. These findings reignite the recent debate over whether imatinib might have utility as a fertility preservation agent in women being treated for cancer with cisplatin (and possibly also other DNA damage inducing chemotherapeutics). ${ }^{5,8,9}$

It is firmly established that TAp63 is essential for DNA damage-induced apoptosis of oocytes. The studies of Suh et al. ${ }^{10}$ and Livera et al. ${ }^{11}$ showed that TAp63 is constitutively expressed in primordial follicle oocytes and that loss of this protein renders these cells profoundly resistant to $\gamma$-irradiation. ${ }^{10,11}$ Subsequent work from Kerr et al. ${ }^{6}$ identified the potent cell killer, Puma, either alone or together with another BH3-only protein, Noxa, as the key downstream mediator/s of TAp63-initiated oocyte death (Figure 1). This revealed that TAp63 and its relative p53 transcriptionally activate the same effectors for inducing apoptosis. ${ }^{6,12,13}$ Kim's in vitro analysis of ovaries from mice with a conditional deletion of p63 in oocytes confirms an essential role for TAp63 in oocyte death following DNA damage. Taken together, these studies demonstrate that TAp63 is responsible for coordinating oocyte death resulting from diverse forms of genotoxic stress.

Interestingly, while TAp63 is proposed to be the master regulator of cisplatin-induced oocyte death, Kim et al. suggest that TAp73 is also an essential player in the oocyte apoptotic signalling cascade, although no data from TAp73-deletion models were provided to prove this. The authors report that expression of both TAp73 and c-Abl was upregulated, while TAp63 expression was downregulated in neonatal mouse ovaries cultured for 2 days in the presence of a dose of cisplatin $(4 \mu \mathrm{M})$ shown to be sufficient to kill $90 \%$ of the oocytes within 4 days in vitro. Furthermore, TAp73 and c-Abl expressions were both reported to be greatly diminished in the absence of p63. Kim et al. therefore suggest that TAp63 initially transcriptionally upregulates the expression of both TAp73 and c-Abl and that post-translational activation of TAp73 by c-Abl then perpetuates the apoptotic cascade via Bax. In contrast to this proposed model, it has previously been shown using pan-p73 as well as TAp73 isoform-specific antibodies that TAp73 is constitutively (i.e., in the absence of a DNA damage inducing agent) expressed in primordial follicle oocytes. ${ }^{11,14}$ Moreover, their model that TAp63 must first transcriptionally induce c-Abl is inconsistent in itself and with a previous report, ${ }^{5}$ which both indicate that $\mathrm{c}$-Abl must posttranslationally modify TAp63 to activate it. Therefore, while the proposed involvement of TAp73 and c-Abl in DNA damage-induced oocyte apoptosis is of interest, at present there are insufficient data to support this premise and future experiments with TAp73 and c-Abl knockout mice would be required to demonstrate a key role for these proteins in this process.

\footnotetext{
${ }^{1}$ Prince Henry's Institute, Clayton, VIC 3168, Australia; ${ }^{2}$ Department of Anatomy and Developmental Biology, Monash University, Clayton, VIC 3168, Australia; ${ }^{3}$ Molecular Genetics of Cancer, The Walter and Eliza Hall Institute of Medical Research, 1 G Royal Parade, Parkville, VIC 3052 , Australia and ${ }^{4}$ Department of Medical Biology, The University of Melbourne, Melbourne, VIC 3000, Australia

${ }^{*}$ Corresponding author: A Strasser, Molecular Genetics of Cancer, The Walter and Eliza Hall Institute of Medical Research, 1G Royal Parade, Parkville, VIC 3050, Australia. Tel: +61 39345 2624; E-mail: strasser@wehi.edu.au
} 
The contribution of p53 to the initiation of oocyte death is also controversial. Previous work failed to immunohistochemically detect p53 in the nuclei of healthy primordial follicle oocytes. ${ }^{11}$ Moreover, although one report indicated that loss of p53 inhibits primordial follicle depletion caused by exposure to the environmental toxicant 9,10-dimethylbenz[a]antracene, ${ }^{15}$ other studies using p53-deficient oocytes demonstrated conclusively that p53 is dispensable for oocyte death following exposure to the chemotherapy drug doxorubicin or $\gamma$-irradiation. ${ }^{3,6,10}$ In contrast to these earlier findings, Kim et al. suggest that, similar to TAp73, p53 expression may also be upregulated by TAp63 in oocytes treated with cisplatin, but the biological significance of this increase in p53 levels for death signalling within the oocyte remains was not established.

In the current study, Kim et al. have also revisited the potential use of imatinib (designed as an inhibitor of BCR-ABL for treatment of CML, but also inhibits C-Abl and indeed certain other kinases) as a fertility preservation agent for women receiving cisplatin anti-cancer treatment, which has recently been a matter of debate..$^{5,8,9}$ In 2009 , Gonfloni et al. ${ }^{5}$ reported that $\mathrm{c}-\mathrm{Abl}$ phosphorylates TAp63 and leads to transcriptional activation of proapoptotic genes, including Bax and Puma, in cisplatin-treated human cancer-derived cell lines. They also reported that imatinib protects mouse oocytes from cisplatininduced death and prolongs fertility in cisplatin-treated mice. Notably, a ferto-protective effect for imatinib could not be demonstrated when these studies were later repeated and extended using two different strains of mice, including longterm breeding studies. ${ }^{8}$ However, in agreement with the former study, Kim and colleagues also suggest that imatinib can prevent cisplatin-induced oocyte death, albeit through preventing c-Abl from activating TAp73, rather than stimulating TAp63, as originally proposed by Gonfloni et al. ${ }^{5}$ Using in vitro ovary cultures, Kim et al. show that while only $\sim 10 \%$ of primordial follicles survive cisplatin treatment, $\sim 50 \%$ of primordial follicles survive during co-culture with cisplatin and imatinib, although the size of the resulting ovaries was small, as seen for treatment with cisplatin alone. The cultured and treated ovaries were then grafted under the kidney capsule of isogenic female mice to provide an in vivo environment, in which to show that oocyte rescue was achieved by imatinib treatment. While surviving follicles were observed 14 days later in the control, imatinib- and imatinib/ cisplatin-treated grafts, tissue was not recovered in grafted ovaries treated with cisplatin alone. These data indicate that in the absence of imatinib, cisplatin treatment completely destroys the ovary. It is somewhat surprising that ovarian tissue, albeit devoid of immature oocytes, was not recovered. This outcome is particularly curious given that it was demonstrated that the $4 \mu \mathrm{M}$ cisplatin dose primarily targets primordial follicles but not the growing follicles or ovarian stromal cells; thus, ample tissue should have been recoverable. As the authors state, it is possible that the impact of cisplatin continues to damage the ovarian tissue, and not just the primordial follicles, after removal of the drug and during the grafting period. Notably, because breeding studies were not possible, the capacity for imatinib to protect against cisplatininduced infertility was not actually tested and thus the fertoprotective effects of imatinib initially reported by Gonfloni et al. ${ }^{5}$ could not be confirmed by Kim and colleagues.

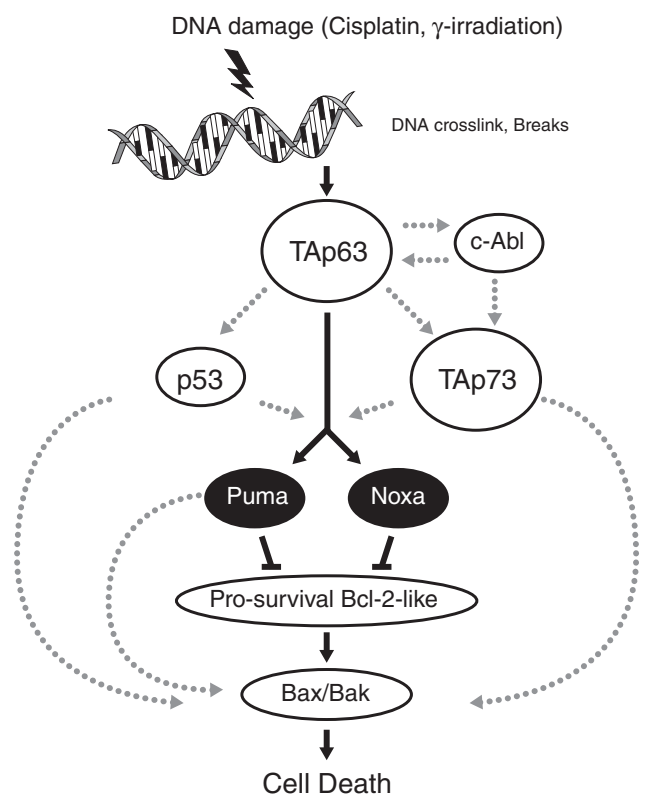

Figure 1 Model to summarise current ideas on the mechanisms by which DNA damage causes the death of primordial follicle oocytes. Processes that are firmly established through experiments with gene-targeted (knock-out) mice are indicated in bold. Processes that have not been proven with such rigour are indicated in lighter font

Given inter-study inconsistencies in the source (Sigma versus hospital grade) and concentration ( 4 versus $20 \mu \mathrm{M}$ ) of cisplatin, as well as the significant impact these parameters appear to have on the ability of imatinib to prevent oocyte death, ${ }^{5,8}$ the clinical utility of imatinib as a ferto-protective adjuvant during anticancer treatment in women remains to be established. It is particularly worrisome that Kim et al. demonstrated imatinib to be toxic to ovaries (follicle somatic cells and oocytes) at doses $>5 \mu \mathrm{M}$, which are within the concentration range found in the plasma of CML patients receiving imatinib treatment (up to $100 \mu \mathrm{M})$. In this regard, follow-up studies on the fertility and endocrine function of women previously treated with imatinib may provide an indication of any potential benefits, or indeed adverse effects, of this chemotherapeutic agent on female fertility and reproductive health.

In conclusion, we believe that at present direct blockade of the proapoptotic $\mathrm{BH}$-only proteins Puma and Noxa or inhibition of their synthesis remains the best-validated strategy to prevent killing of primordial follicle oocytes and thus preserve fertility in women subjected to DNA damage inducing anti-cancer therapeutics (Figure 1).

1. Meirow D et al. Clini Obstet Gynecol 2010; 53: 727-739.

2. Jeruss JS, Woodruff TK. N Engl J Med 2009; 360: 902-911.

3. Perez Gl et al. Nat Med 1997; 3: 1228-1232.

4. Kujjo LL et al. PLoS One 5: e9204.

5. Gonfloni $S$ et al. Nat Med 2009; 15: 1179-1185.

6. Kerr JB et al. Mol Cell 2012; 48: 343-352

7. Kim et al. Cell Death Differ 2013; 20: 987-997.

8. Kerr JB et al. Nat Med 2012; 18: 1170-1172; author reply 1172-1174.

9. Maiani E et al. Nat Med 2012; 18: 1172-1174.

10. Suh EK et al. Nature 2006; 444: 624-628.

11. Livera G et al. Reproduction 2008; 135: 3-12.

12. Villunger $A$ et al. Science 2003; 302: 1036-1038.

13. Jeffers JR et al. Cancer Cell 2003; 4: 321-328.

14. Tomasini R et al. Genes Dev 2008; 22: 2677-2691.

15. Pru JK et al. Reprod Sci 2009; 16: 347-356. 